\title{
Sudden unexpected death in epilepsy (SUDEP): a clinical perspective and a search for risk factors
}

\author{
Robert Kloster, Torstein Engelskjøn
}

\begin{abstract}
Objectives-To examine the risk factors and their relative importance and possible role in sudden unexpected death in epilepsy (SUDEP).

Methods-The study was conducted as a retrospective analysis of deaths in an outpatient population of a tertiary referral centre, based on clinical and pathological data.

Results-Of a total of 140 deaths, 61 (44\%) had not been to postmortem and were excluded, $37(26 \%)$ had a verified cause of death and formed the non-SUDEP group, and $42(30 \%)$ were classified as SUDEP. In the SUDEP group there was pulmonary oedema in $62 \%$, signs of preceding seizures in $67 \%$, no visible seizures in three of six observed deaths. A high seizure frequency prevailed in SUDEP as well as non-SUDEP. Sixty per cent of deaths were sleep related. Various other circumstances were temporally associated with death. The prone position at death was seen in $71 \%$ of the SUDEP patients; possible interpretations are discussed. Supposedly subtherapeutic serum concentrations of one or more antiepileptic drugs were found in $57 \%$ of those with reported serum concentrations. Alcohol was not a factor in the material, whereas hyponatraemia was seen in two cases.

Conclusions-Most cases of SUDEP are preceded by seizures; their presence, frequency, type, aetiology, tractability, and the use of antiepileptic drugs are factors in the demise. No common risk factor, present in all cases of SUDEP, could be found, suggesting the probability of multiple mechanisms behind SUDEP.

(F Neurol Neurosurg Psychiatry 1999;67:439-444)
\end{abstract}

Keywords: sudden death in epilepsy; risk factors

The National Center for Epilepsy, Sandvika, Norway

R Kloster

T Engelskjøn

Correspondence to: Dr Robert Kloster, The National Center for Epilepsy, PO Box 900, N-1301

Sandvika, Norway.

Telephone 004767554000 fax 004767545321 .

Received 11 June 1998 and in final form

8 March 1999

Accepted 18 March 1999
Sudden unexpected death in epilepsy (SUDEP) is a major diagnostic category in studies of mortality in epilepsy, accounting for an incidence of $1 / 1000$ to $1 / 100$, depending on the cohort studied. ${ }^{1}$ Among risk factors identified are presence of seizures, poor seizure control, chronic refractory epilepsy, male sex, subtherapeutic concentrations of antiepileptic drugs, and the influence of sleep as well as alcohol. No risk factor common to all cases of SUDEP has been found.

The mechanism of SUDEP is not fully understood. Seizures usually start the final event. White et $a l^{2}$ have described the effect of seizures in human volunteers with epilepsy, and found marked changes in cerebral dynamics with an increase in CSF pressure to more than $1000 \mathrm{~mm} \mathrm{H}_{2} \mathrm{O}$. Pressure rise is tolerated until it approaches diastolic pressure, impairing normal cerebral circulation. ${ }^{3}$ Most seizures are accompanied by apnoea and a fall in oxygen tension in humans and animal models alike. ${ }^{45}$ Johnston et al studied an animal model and concluded that hypoventilation is an important cause of death during seizures. ${ }^{5}$

The present study is a retrospective investigation of deaths in a population with chronic refractory epilepsy, with an emphasis on risk factors and their relative importance in SUDEP.

\section{Methods}

We adopted the definition of SUDEP proposed by Nashef: "Sudden unexpected, witnessed or unwitnessed, nontraumatic and nondrowning death in patients with epilepsy, with or without evidence for a seizure and excluding documented status epilepticus, in which postmortem examination does not reveal a toxicologic or anatomic cause for death". ${ }^{6}$

"Sudden" means in a matter of minutes or hours after the onset of the final ictus or event. Unexpected means that the subjects were in a reasonable state of health before the final event. ${ }^{6}$

We examined 140 patients with epilepsy who died between 1965 and 1996. Most had been outpatients of a tertiary referral centre in the past or at the time of death. Most had chronic refractory epilepsy. They came from different parts of Norway, but do not constitute a complete list of deaths in a circumscribed population, and are not conducive to studies of incidence. Information was collected from medical histories in our hospital as well as from local hospitals, local doctors, family members, police, and postmortem reports. All data in this report are based on statements from one or more of these sources.

All patients included in the present study were necropsied, most of them in university departments of pathology. Each brain was examined by pathologists with a special interest

Table 1 Demography and time relations (mean values)

\begin{tabular}{llll}
\hline & SUDEP & non-SUDEP & p Value \\
\hline Males & $26(62 \%)$ & $19(51 \%)$ & \\
& & & 0.34 \\
Females & $16(38 \%)$ & $18(49 \%)$ & \\
$\begin{array}{l}\text { Age at epilepsy onset (y) } \\
\text { (SD) }\end{array}$ & 8.2 & 12.7 & 0.04 \\
$\begin{array}{l}\text { Age at death (y) } \\
\text { (SD) }\end{array}$ & $\begin{array}{l}(8.6) \\
(1.9\end{array}$ & 32.6 & 0.31 \\
$\begin{array}{l}\text { Duration of epilepsy (y) } \\
\text { (SD) }\end{array}$ & $\begin{array}{l}(19.8 \\
(14.1)\end{array}$ & $\begin{array}{l}(19.0) \\
(14.9)\end{array}$ & 0.94 \\
\hline
\end{tabular}


Table 2 Main postmortem findings: brain, heart, and lungs

\begin{tabular}{|c|c|c|c|c|c|c|}
\hline & \multicolumn{2}{|l|}{ Brain } & \multicolumn{2}{|l|}{ Heart } & \multicolumn{2}{|l|}{ Lungs } \\
\hline & $S U D E P$ & non-SUDEP & $S U D E P$ & non-SUDEP & $S U D E P$ & non-SUDEP \\
\hline Normal & 12 & 6 & 28 & 23 & 11 & 3 \\
\hline Oedema or congestion & 8 & 3 & 3 & 0 & 26 & 10 \\
\hline Old change, non-fatal & $20^{\star}$ & $20 \dagger$ & 115 & 69 & $4+t$ & $5 \neq$ \\
\hline Recent change, fatal & 0 & $2 \ddagger$ & 0 & $7 \star \star \star$ & 0 & $17^{55}$ \\
\hline Terminal changes or no data & 2 & 6 & 0 & 1 & 1 & 2 \\
\hline Sum & 42 & 37 & 42 & 37 & 42 & 37 \\
\hline
\end{tabular}

Brain: ${ }^{\star}$ malformation four; traumatic or surgical six, hypoxic 10 ; †malformation four, traumatic or surgical five; hypoxic 11 ; $\ddagger$ traumatic one, mitochondrial encephalopathy one.

Heart: $\int$ atherosclerosis three; fibrosis three, various five; $\lceil$ atherosclerosis three, various three; $\star \star$ infarction two, myocarditis two, various three.

Lungs: ††emphysema one, various three; łłaspiration two, various three; \$Spneumonia 12, aspiration two, drowning two, carcinoma one.

in neuropathology, whereas the hearts and lungs were examined by general pathologists. Thirty seven patients with a defined cause of death constitute the non-SUDEP group. Forty two patients who fulfilled the criteria of our definition are included in the SUDEP group. No necropsy was performed in 61 patients and these were excluded from the study. Postmortem serum concentration analyses were carried out at the National Institute for Forensic Toxicology in Oslo, Norway. Statistical treatment of SUDEP versus non-SUDEP patients is based on Student's $t$ test or Pearson's $\chi^{2}$ test. Certain data - for example, measurement of postmortem serum concentrations-were incomplete, rendering comparisons inconclusive.

\section{Results}

On comparing the 42 patients with SUDEP with the 37 non-SUDEP patients (table 1), no significant differences were found for sex, age at death, or duration of epilepsy. Age at onset of epilepsy was significantly lower in the SUDEP group than in the non-SUDEP group $(\mathrm{p}=0.04)$.

\section{POSTMORTEM FINDINGS}

The pathological findings disclosed at postmortem did not explain death in any of the 42 SUDEP patients, whereas in the 37 nonSUDEP patients causes such as pneumonia, cardiac disease, status epilepticus, trauma, suicide, and drowning were found.

\section{Brain}

Pathological changes in the brain or brain stem were present in 30 of 42 SUDEP patients $(71 \%)$. Two patients with cerebral oedema had minor tentorial impressions, but there was no structural damage to the brain stem, ventricular compression, or compression of gyri (table 2).

Among 37 non-SUDEP patients, only six had a structurally normal brain. The remaining $31(84 \%)$ showed pathological changes, and two were considered fatal. Aetiologies of epilepsy (ICD-10), ${ }^{7}$ taking into account clinical, neuroimaging, and neuropathological findings, were assessed as 15 symptomatic and 27 idiopathic in the SUDEP group; 21 symptomatic and 16 idiopathic in the non-SUDEP group $(\mathrm{p}=0.008)$.
Heart (table 2).

Pathological changes in the heart, which were all presumed to be non-fatal, were described in $33 \%$ of the SUDEP patients. In one patient a special investigation of the conduction system of the heart disclosed fibrosis and oedema of the conductive tissue. Thirty eight per cent of the non-SUDEP group showed pathological changes of the heart; fatal in at least seven patients.

\section{Lungs}

There were marked differences between the groups, with pulmonary oedema in $62 \%$ of SUDEP patients and $27 \%$ of non-SUDEP patients, as well as various other pulmonary conditions in the second group (table 2).

SEIZURES

There was a significant difference in seizure types between the groups, with more primarily generalised seizures in the SUDEP group $(p=0.03)$. All SUDEP patients had generalised motor seizures (19 primarily, 21 secondarily generalised, and two unclassified). Generalised motor seizures also prevailed in the nonSUDEP group, but $5 \%$ had only partial seizures $(p=0.07)$.

In the SUDEP group, signs of seizures immediately before death in $67 \%$ (table $3 \mathrm{~A}$ ) included observed seizures, fresh bites, blood on the pillow, and cyanosis, as well as reported sounds of ongoing seizures. Such signs are suggestive, if not conclusive, of generalised motor seizures. The great majority in both groups had a high seizure frequency with more than

Table 3 Presence of seizures

(A) Signs of seizure preceding death

\begin{tabular}{|c|c|c|}
\hline & SUDEP & Non-SUDEP \\
\hline Positive signs & 28 & 13 \\
\hline No sign & 11 & 10 \\
\hline No data & 3 & 14 \\
\hline \multicolumn{3}{|c|}{$\begin{array}{l}\text { Significant difference between SUDEP and non-SUDEP } \\
(p=0.002)\end{array}$} \\
\hline \multicolumn{3}{|c|}{$\begin{array}{l}\text { (B) Seizure frequency } \\
\text { (high: }>12 \text { seizures } / y \text {, intermediate: } 2-12 / y \text {, low: } \leqslant 1 / y \text { ) }\end{array}$} \\
\hline & $S U D E P$ & Non-SUDEP \\
\hline High & 32 & 26 \\
\hline Intermediate & 3 & 1 \\
\hline Low & 4 & 6 \\
\hline No data & 3 & 4 \\
\hline
\end{tabular}

No significant difference between SUDEP and non-SUDEP $(\mathrm{p}=0.56)$ 
Table 4 Six witnessed cases of sudden, unexpected death in epilepsy

\begin{tabular}{lllllll}
\hline \multicolumn{2}{c}{ Patient No } & Preceding event & Time span & Seizure & Time span before death & Relevant pathology \\
\hline A & 18 & Exercise & 1 hour & GTC & Failed resuscitation & None \\
& 24 & None & Immediate & GTC & Failed resuscitation & Pulmonary oedema \\
& 257 & Exercise & Immediate & GTC & Failed resuscitation & Terminal changes \\
B & 25 & Minor surgery & 12 hours & None & Immediate & Pulmonary oedema \\
& 26 & None & - & None & Immediate & Cerebello-olivar degeneration \\
& 40 & None & - & None & Immediate & None
\end{tabular}

$\star$ Uneventful removal of mandibular exostosis.

A: with seizures (three cases). B: without seizures (three cases). GTC: generalised tonic-clonic seizure.

Table 5 Position at death (24 patients with a reported position)

\begin{tabular}{lll}
\hline & SUDEP & $\%$ \\
\hline Prone position & 17 & 71 \\
Supine position & 1 & 4 \\
Other position & 6 & 25 \\
\hline
\end{tabular}

Preponderance of prone position in SUDEP, $p=0.001$

Table 6 Choice of antiepileptic drugs (AEDs)

\begin{tabular}{lll}
\hline & SUDEP & non-SUDEP \\
\hline Phenobarbital, primidone & 17 & 18 \\
Phenytoin & 10 & 13 \\
Carbamazepine & 20 & 15 \\
Oxcarbazepine & 6 & 3 \\
Valproate & 9 & 11 \\
Benzodiazepines & 5 & 8 \\
Other AEDs & $3^{\star}$ & $3 \dagger$ \\
Unknown & 0 & 1 \\
Just discontinued AED & 2 & 2 \\
No AED treatment & 0 & 1 \\
\hline
\end{tabular}

^Ethotoin (one), lamotrigine (one), vigabatrin (one).

†Vigabatrin (two), ethosuximide (one).

12/year (table $3 \mathrm{~B}$ ), as could be expected in a cohort with chronic refractory epilepsy. A precise correlation between seizure type and seizure frequency was not possible. Of six observed deaths in the SUDEP group (table 4), three had generalised motor seizures and three had no clinical seizures.

\section{OTHER CIRCUMSTANCES}

Twenty five of the SUDEP patients were found in bed, presumably dying before, during, or after sleep. Assuming 8 hours of sleep/24 hours, only 14 patients would be expected to have died in relation to sleep. Because many of the non-SUDEP cases died in hospital or in an accident, it is not meaningful to relate their deaths to the sleep-wake cycle. Various somatic conditions unrelated to epilepsy, and not life threatening, were reported among $47 \%$ of SUDEP patients. In the non-SUDEP group, $23(62 \%)$ had a somatic disease with a fatal outcome.

There were 10 cases of mental retardation and two of dementia in the SUDEP group; in the non-SUDEP group there were 11 mentally

Table 7 Postmortem serum concentrations of antiepileptic drugs (AEDs)

\begin{tabular}{lll}
\hline & SUDEP & non-SUDEP \\
\hline No AEDs detected & 0 & $2 \dagger$ \\
All in recommended range & 9 & 4 \\
One in recommended range, one supposedly subtherapeutic & 9 & 0 \\
All subtherapeutic & 4 & 0 \\
Toxic & $1^{\star}$ & $3 \ddagger$ \\
\hline
\end{tabular}

^Moderately raised phenytoin $(150 \mu \mathrm{mol} / \mathrm{l})$ :not lethal

†One without AEDs, one recently discontinued carbamazepine.

¥Two cases of lethal phenobarbital (440 resp. $800 \mu \mathrm{mol} / \mathrm{l}$ ); one case of potentially lethal carbamazepine $(275 \mu \mathrm{mol} / 1)$ : due to interaction with erythromycin. retarded patients, three demented patients, and one patient with Wernicke's encephalopathy $(\mathrm{p}=0.40)$.

\section{POSITION AT DEATH}

We define the "prone position" as lying on the belly, chest, or face, with or without obstruction of the nose or mouth. The "supine position" is defined as lying on the back, with no obstruction of the nose or mouth.

Position at death was reported in 24 SUDEP patients (table 5). Seventeen cases ( $40 \%$ of all) were found in the prone position, and only one $(2 \%)$ in the supine position, whereas six $(14 \%)$ were found in other positions. Body position in the remaining 18 of 42 SUDEP patients was not recorded.

Considering only those with a verified position (table 5), $71 \%$ were lying prone, $4 \%$ supine, and $25 \%$ in other positions. Assuming an equal likelihood of either the prone or the supine positions, this difference was significant $(\mathrm{p}=0.001$; two tailed test).

TREATMENT WITH ANTIEPILEPTIC DRUGS

A small excess of carbamazepine or oxcarbazepine treatment in the SUDEP group (table 6) is not amenable to statistical analysis, because of polytherapy and possible drug interactions. There were no significant differences between the groups for monotherapy or polytherapy $(\mathrm{p}=0.10)$.

Serum concentrations of antiepileptic drugs were analysed in 23 of the SUDEP patients (table 7). Of these, 39\% had drug concentrations in the recommended range and $57 \%$ had supposedly subtherapeutic concentrations of one or more antiepileptic drugs.

Five of the SUDEP patients used neuroleptic or antidepressant drugs and two used cardiopulmonary drugs. Corresponding figures for the non-SUDEP group were five and three. No cases of street drugs were discovered.

BLOOD ALCOHOL

In 28 SUDEP cases examined for blood alcohol postmortem, there was a low ethanol concentration in only one, and none in the non-SUDEP group.

\section{HYPONATRAEMIA}

Two cases of hyponatraemia were found in the SUDEP group. One was based on serum sodium measured at necropsy and associated with the syndrome of inappropriate antidiuretic hormone secretion (SIADH), ${ }^{8}$ the other on serum sodium measurement 1 month before death. Both were associated with the use 
of oxcarbazepine; one had recently changed from carbamazepine. Serum sodium values before death or at necropsy were missing for the remaining cases presented here.

\section{Discussion}

Our material comes from a selected group with chronic refractory epilepsy. It contained a high percentage of SUDEP cases (30\% of the total). Other studies report up to $50 \%^{1}$; highest in cohorts with intractable epilepsy. There were no significant demographic differences between SUDEP and non-SUDEP groups, except for a slightly lower age at epilepsy debut in SUDEP and a non-significant excess of males. A male dominance in SUDEP has been found in other studies. ${ }^{9}$

By definition, no adequate explanation of death was found at necropsy, but pathological changes were found in various organs. The most consistent finding was oedema of the lungs, often haemorrhagic. In an experiment on sheep, Johnston et al found evidence that central hypoventilation had a role in the aetiology of sudden unexpected death and confirmed the association with pulmonary oedema, but they did not think that the development of hypoventilation could be due to pulmonary oedema. ${ }^{5}$

Cardiac pathology was found in 14 patients in both groups. Three SUDEP patients showed fibrosis localised in the conduction system, around the atrioventricular bundle, or diffusely in the myocardium. Seizure related ischaemia or hypoxic changes have been suggested as a possible explanation, ${ }^{10-12}$ rendering the heart vulnerable to subsequent epileptic seizures.

In both groups, a large proportion had pathological changes in the brain. No predilection could be found with respect to type and location of pathology. Oedema was seen more often in the SUDEP group, but there was no evidence of mass effect or cerebral herniation. Thirty SUDEP patients had structural brain lesions versus 31 in the non-SUDEP group. Some of these changes provide an aetiology for the epilepsy-for example, cortical dysgenesis or tuberous sclerosis. Most of the changes imply neurological deficit of a static nature, and provide no obvious explanation for the final event.

There were signs of seizures starting the final event in $67 \%$ of SUDEP patients, all suggestive of generalised motor seizures. This is a minimal figure, as not all seizures can be detected. In our material of six observed deaths three were associated with clinical seizures and in three patients no seizures were seen. Among a total of 12 witnessed SUDEP patients three were not associated with visible seizures. ${ }^{1314}$ Leestma et al and Nashef et al found evidence of seizures in $50 \%$ and $64 \%$, respectively, of SUDEPs. ${ }^{15}{ }^{16}$ Subclinical epileptic activity may be an explanation, as pointed out by Lathers $e t$ $a .^{17}$

Most of our patients had relatively high seizure frequency, with more than 12/year, mainly generalised motor seizures. Consistent with epidemiological studies, there is a high percentage of SUDEP in our material, which shows that cohorts with high seizure frequency have a relatively high risk of sudden death, whereas the opposite is the case for those with few seizures. ${ }^{18}$ In our study, however, there was no difference in seizure frequency between the SUDEP and non-SUDEP groups to support this finding. The expected low seizure frequency in the non-SUDEP group was not found because our patients were selected on the basis of poor seizure control, and patients with low seizure frequency are few in both groups.

Clearly, seizures play an important part in SUDEP, but some patients die without visible seizures. We must consider the possibility that some of these deaths are unrelated or only indirectly related to epilepsy. The incidence of sudden cardiac death due to ischaemic heart disease is increased in people with epilepsy. ${ }^{19}$ Examples of seizure related changes in the heart have been given by White $e t$ al, ${ }^{2}$ Natelson et $a l,{ }^{12}$ and Mosier et al. ${ }^{20}$ Acute symptoms may not be registered if the patients die alone, and ischaemic changes in the heart may not be visible at necropsy unless they survive for more than 2 hours. Such cases may be difficult to diagnose. We have included the case of a 40 year old woman with epilepsy who had moderate chest pain, normal ECG, and was found dead in the daytime, having urinated, without coronary obstruction at necropsy. We cannot establish the cause of death with any degree of certainty and have included her in the SUDEP group.

Cardiac arrhythmias may be primary or they can be triggered by epileptogenic activity, ${ }^{17}$ and sometimes, although rarely, by carbamazepine. ${ }^{21}$ Withdrawal of antiepileptic drugs (carbamazepine or phenytoin) may also influence the cardiac activity and, possibly, cause sudden death. ${ }^{22}$ Prolonged QT time is probably related to sudden death and life threatening arrhythmias. ${ }^{23}$ Accordingly, we cannot distinguish reliably between sudden deaths related to epilepsy and some other causes, sudden cardiac death being the predominant category. Most SUDEP material is likely to include deaths unrelated to epilepsy.

Seventy one per cent of patients were found dead in the prone position, $4 \%$ in the supine position, and $25 \%$ in other positions. Assuming an equal chance of either the prone or the supine position, a simple probability test shows that the difference is significant $(\mathrm{p}=0.001)$. Earnest et al found $81 \%$ of SUDEP cases in the prone position. ${ }^{14}$ Coyle et al report $58 \%$ prone of those with a reported position. ${ }^{24}$ Nashef et al report 11 of 26 in which the position of the head was such that breathing could have been compromised. ${ }^{25}$ Position may influence ventilation in several ways. The supine position may affect ventilation in an unconscious patient because of the gravitational pull on the tongue and other mechanisms, as has been demonstrated in obstructive sleep apnoea. ${ }^{26-28}$ The prone position may cause obstruction of the nose and mouth due to pressure against the bed clothing. Changing the sleeping position from prone to supine has brought about a marked reduction in the incidence of the 
sudden infant death syndrome. Several explanations have been suggested, some of them possibly relevant also for the discussion of SUDEP - for example, the influence of flexion or extension of the cervical spine on the patency of the upper airways. ${ }^{29}$ Clearly, both the reporting of position and the interpretation of findings are inadequate in the current literature.

In the SUDEP group, 58\% were found dead in bed, presumably dying in relation to sleep. This may be related to the horizontal position as described, or it may be due to sleep itself. Control of sleep is closely related anatomically and functionally to respiratory control. ${ }^{30-32}$ During sleep, respiration is dependent on the inherent rhythmicity of the metabolic and automatic respiratory control system located in the medulla oblongata. ${ }^{31}{ }^{32}$ Central sleep apnoea syndromes are characterised by cessation of rhythmical breathing during sleep, sometimes with a fatal outcome. ${ }^{30-33}$ Sullivan et al use the term central sleep apnoea as a general description for sleep induced failure of the neural elements that control breathing. ${ }^{32}$ Epileptic seizures are also associated with central apnoea and sometimes death, often occurring during sleep.

Death occurred in direct relation to physical exertion in four patients, two of them in the SUDEP group, all apparently from precipitating seizures. This relation has been pointed out by Hirsch and Martin. ${ }^{13}$

We found more use of carbamazepine and oxcarbazepine in the SUDEP group than in the non-SUDEP group, but the difference in antiepileptic drug use was not amenable to statistical analysis. Timmings found SUDEP patients significantly more likely to be taking carbamazepine. ${ }^{34}{ }^{35}$ Others have found no specific pattern of antiepileptic drug use in SUDEP. ${ }^{96}$ Monotherapy versus polytherapy does not seem to be a factor in our study. Leestma $e t a l^{15}$ and Timmings ${ }^{35}$ made similar findings, whereas Derby et $a l^{37}$ found that subjects taking two or more antiepileptic drugs may be at a somewhat increased risk of SUDEP.

Supposedly subtherapeutic concentrations of one or more antiepileptic drugs have been found in $57 \%$ of SUDEP patients; $77 \%$ had concentrations in the recommended range of one or more antiepileptic drugs. Other studies report similar findings $s^{15} 38-40$

Other drugs could play a part in sudden death due to toxicity, interactions, and lowering of the seizure threshold, but comedication did not differ appreciably between our groups. Tennis et al found that the incidence of SUDEP increased with prescription of psychotropic drugs. ${ }^{41}$ Suicidal use of drugs is a relatively frequent cause of sudden death, and should be diagnosed by a complete toxicological investigation.

Three cases of hyponatraemia have been recorded among SUDEP patients known to us, two of them in the SUDEP group presented here; all on oxcarbazepine medication. Two of these cases were associated with SIADH,,${ }^{42-44}$ and are presented separately. ${ }^{8}$ Hyponatraemia and SIADH have been associated with other drugs and pathological conditions. ${ }^{44}$

\section{Conclusions}

Most cases of SUDEP are preceded by seizures. Their presence, frequency, type, triggering factors, and individual tractability with antiepileptic drugs are factors in the demise. No risk factor common to all cases of SUDEP could be found, suggesting the probability of multiple mechanisms behind SUDEP.

We are indebted to the following persons and institutions, all in Oslo, Norway, for placing data at our disposal and providing advice: Professor Ansgar Torvik at the Laboratory of Pathology, Oslo City Hospital; Professor Kari Skullerud at the Section for Neuropathology, The National Hospital; Professor Bjørnar Olaisen at the Institute of Forensic Medicine; The National Olaisen at the Institute of Forensic Medicine; The National
Institute of Forensic Toxicology, and to Ms Inger Vågen for assistance with the technical preparation of the manuscript.

1 Nashef L, Sander JWAS. Sudden unexpected deaths in epilepsy: where are we now? Seizure 1996;5:235-8.

2 White PT, Grant P, Mosier J, et al. Changes in cerebral dynamics associated with seizures. Neurology 1961;11:35461.

3 Plum F, Posner JB. The diagnosis of stupor and coma. In: Plum F, McDowell FH, eds. Contemporary neurology series 5. Oxford: Blackwell, 1966:1-197.

4 Nashef L, Walker F, Allen P, et al. Apnoea and bradycardia during epileptic seizures: relation to sudden death in epilepsy. $尹$ Neurol Neurosurg Psychiatry 1996;60:297-300.

5 Johnston SC, Horn JK, Valente J, et al. The role of hypoventilation in a sheep model of epileptic sudden death. Ann Neurol 1995;37:531-7.

6 Nashef L. Sudden unexpected death in epilepsy: terminology and definitions. Epilepsia 1997;38(suppl 11):S6-8.

7 World Health Organization. ICD-10. International statistical classification of diseases and related health problems. Geneva: WHO, 1992;1:1-1243.

8 Kloster R, Børresen HC, Hoff-Olsen P. Sudden death in two patients with epilepsy and the syndrome of inappropriate antidiuretic hormone secretion (SIADH). Seizure 1998;7: $1-2$.

9 Leestma JE, Annegers JF, Brodie MJ, et al. Sudden unexplained death in epilepsy: observations from a large clinical development program. Epilepsia 1997;38:47-55.

10 Falconer B, Rajs J. Post-mortem findings of cardiac lesions in epileptics: a preliminary report. Forensic Sci 1976;8:6371.

11 Tigaran S, Rasmussen V, Dam M, et al. Do epileptic seizures induce myocardial damage leading to sudden death? Epilepsia 1996;37(suppl 5):38.

12 Natelson BH, Suarez RV, Terrence CF, et al. Patients with epilepsy who die suddenly have cardiac disease. Arch Neurol 1998;55:857-60.

13 Hirsch CS, Martin DL. Unexpected death in young epileptics. Neurology 1971;21:682-90.

14 Earnest MP, Thomas GE, Eden RA, et al. The sudden unexplained death syndrome in epilepsy: demographic, clinical, and postmortem features. Epilepsia 1992;33:3106.

15 Leestma JE, Walczak T, Hughes JR, et al. A prospective study on sudden unexpected death in epilepsy. Ann Neurol 1989;26:195-203.

16 Nashef L, Garner S, Fish D, et al. Circumstances surrounding sudden death in epilepsy: interviews with relatives. Epilepsia 1994;35(suppl 7):18

17 Lathers CM, Schraeder PL, Weiner FL. Synchronization of cardiac autonomic neural discharge with epileptogenic activity: the lockstep phenomenon. Electroencephalogr Clin Neurophysiol 1987;67:247-59.

18 Nashef L, Fish DR, Garner S, et al. Sudden death in epilepsy: a study of incidence in a young cohort with epilepsy: a study of incidence in a young cohort with

19 Annegers JF, Hauser WA, Shirts SB. Heart disease mortality and morbidity in patients with epilepsy. Epilepsia 1984;25: 699-704.

20 Mosier JM, White P, Grant P, et al. Cerebroautonomic and myographic changes accompanying induced seizures. Neurology 1957;7:204-10.

21 Dreifuss FE, Mattson RH, Meldrum BS, et al. Antiepileptic drugs. 3rd ed. New York: Raven Press, 1989.

22 Kennebäck G, Ericson $M$, Tomson $T$, et al. Changes in arrhythmia profile and heart rate variability during abrupt withdrawal of antiepileptic drugs. Implications of sudden death. Seizure 1997;6:369-75.

23 Amlie J P. QT dispersion and sudden cardiac death. Eur Heart $\mathcal{7}$ 1997; 18:189-90.

24 Coyle HP, Baker-Brian N, Brown SW. Coroners' autopsy reporting of sudden unexplained death in epilepsy reporting of sudden unexplained death
(SUDEP) in the UK. Seizure 1994;3:247-54.

25 Nashef L, Garner S, Sander JWAS, et al. Circumstances of death in sudden death in epilepsy: interviews of bereaved death in sudden death in epilepsy: interviews of bereaved
relatives. $\mathcal{F}$ Neurol Neurosurg Psychiatry 1998:64:349-52. 
26 Ono T, Lowe AA, Ferguson KA, et al. Associations among upper airway structure, body position, and obesity in skeletal class I male patients with obstructive sleep
f Orthod Dentofacial Orthop 1996;109:625-34

27 Pae E-K, Lowe AA, Sasaki K, et al. A cephalometric and electromyographic study of upper airway structures in the upright and supine positions. Am $\mathcal{F}$ Orthod Dentofacial Orthop 1994;106:52-9.

28 Partinen M, Guilleminault C, Quera-Salva M-A, et al. Obstructive sleep apnoea and cephalometric roentgenograms. Chest 1988;93:1199-205.

29 Schatz A. The significance of anatomohistological findings in the upper airway of SIDS victims. In: Rognum TO, ed. Sudden infant death syndrome. New trends in the nineties. Oslo: Scandinavian University Press, 1995:203-6.

30 Chokroverty S. Sleep and breathing in neurological disorders. In: Edelman NN, Santiago TV, eds. Breathing disorders of sleep. Churchill Livingstone, 1986:225-64.

31 Chokroverty S. The assessment of sleep disturbance in autonomic failure. In: Bannister R, Mathias CJ, eds. Autonomic failure. 3rd ed. Oxford University Press, 1992:442nomic

32 Sullivan CE, Issa FG, Berthon-Jones M, et al. Pathophysiology of sleep apnea. In: Saunders BA, Sullivan CE, eds. Sleep and breathing. New York: Marcel Dekker, 1984:299363.

33 Deveraux MV, Keane JR, Davis RL. Automatic respiratory failure associated with infarction of the medulla. Arch Neurol 1973;29:46-52.

34 Timmings PL. Sudden unexpected death in epilepsy: a local audit. Seizure 1993;2:287-90.
35 Timmings PL. Sudden unexpected death in epilepsy: is carbamazepine implicated? Seizure 1998;7:289-91.

36 Leppik IE, So EL, Hauser WA, et al. Prospective study of death in epilepsy. Epilepsia 1995;36(suppl 3):S224.

37 Derby LA, Tennis P, Jick H. Sudden unexplained death among subjects with refractory epilepsy. Epilepsia 1996;37: $931-5$.

38 Lund A, Gormsen $\mathrm{H}$. The role of antiepileptics in sudden death in epilepsy. Acta Neurol Scand 1985;72:444-6.

39 Tomson T, Sköld A-C, Holmgren P, et al. Postmortem changes in blood concentrations of phenytoin and carbamazepine: an experimental study. Therapeutic Drug Monitoring 1998;20:309-12.

40 Schnabel R, May Th, Rambeck B. Postmortem determination of anticonvulsant concentrations and lung oedema in the sudden, unexpected death of epileptics. Seizure 1997;6: $327-8$.

41 Tennis P, Cole TP, Annegers JF, et al. Cohort study of incidence of sudden unexplained death in persons with seizure disorder treated with antiepileptic drugs in Saskatchewan, Canada. Epilepsia 1995;36:29-36.

42 Arieff AI, Ayus JC, Fraser CL. Hyponatremia and death or permanent brain damage in healthy children. BMF 1992;304:1218-22.

43 Arieff AI. Hyponatremia, convulsions, respiratory arrest, and permanent brain damage after elective surgery in healthy women. $N$ Engl f Med. 1986;314:1530-5.

44 Spigset $\mathrm{O}$, Hedenmalm K. Hyponatremia and the syndrome of inappropriate antidiuretic hormone secretion (SIADH) induced by psychotropic drugs. Drug Saf 1995;12:209-25. 